\title{
Combining Ability Analysis , Estimation of Heterosis and Some Genetic Parameters Using Half Diallel Cross in Bread wheat (Triticum aestivum L.)
}

\author{
Mohammed H. Ayoob \\ Department of Biology, College of Education for pure sciences, University of Mosul \\ Email: $\underline{\text { mhayoob@uomosul.edu.iq }}$
}

(Received January 31, 2019; Accepted May 16, 2019; Available online March 01, 2020)

DOI: 10.33899/edusj.2020.164365, () 2020, College of Education for Pure Science, University of Mosul. This is an open access article under the CC BY 4.0 license (http://creativecommons.org/licenses/by/4.0/).

\begin{abstract}
:
Five varieties of bread wheat (Triticum aestivum L.) Tamuz-3 Adnaniya, Abu-Greebe-3, IPA99 and Sham-6 and their half diallel crosses were used to study general and specific combining ability for plant height, number of tillers per plant, number of spikes per plant, spike length , number of grains per spike, 100 grains weight and grain yield per plant. The result of the analysis of variance showed that the mean square of genotypes, (GCA) and (SCA) were highly significant for all studied characters. The ratio between the component variance of (GCA) and the component of variance (SCA) was less than one for all studied characters except for plant height which was more than one this indicate the importance of additive and dominance gene effects for all characters, but the dominance gene effects is more important for determining these characters. The largest and positive value for (GCA) effect was in the following parental varieties : Abu- Ghreeb-3 for plant height and number of grains per spike, IPA-99 for number of tillers and number of spikes per plant, Adnaniya for spike length and 100 grains weight and grain yield per plant. The (SCA) effects value in some hyprids was positive with desirable direction, but other hyprids showed negative with undesirable direction for all studied characters therefor heterosis can be utilized for improve these characters. The hyprids $(1 \times 3),(1 \times 4)$, $(2 \times 3),(2 \times 4),(2 \times 5),(4 \times 5)$ revealed desirable and high significant heterosis for all studied characters. The broad sense heritability were high for all studied, however the narrowsense heritability were high for plant height and medium to remained characters. There were over dominance for all characters except partial dominance for plant height.
\end{abstract}

Keywords: Diallel cross, Combining ability, Heterosis, Heritability, Average degree of dominance , Common wheat.

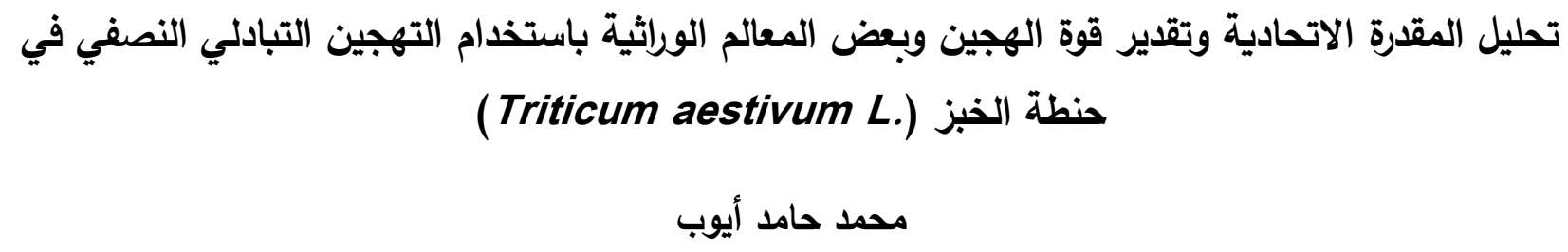

قسم علوم الحياة, كلية التربية للعوم الصرفة, جامعة الموصل, الموصل, العراق 


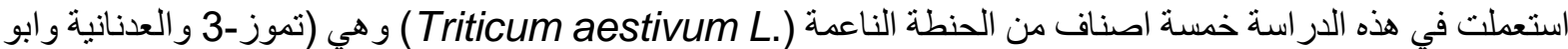

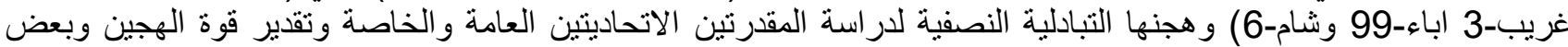

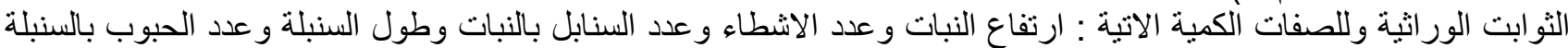

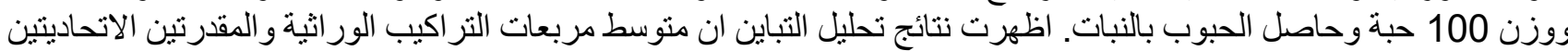

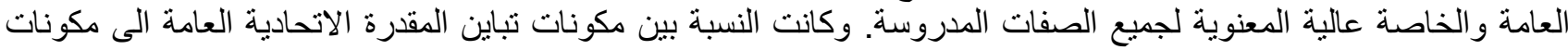

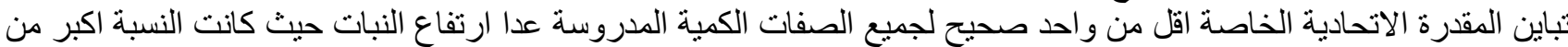

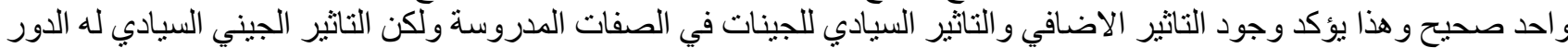

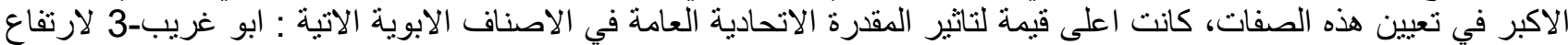

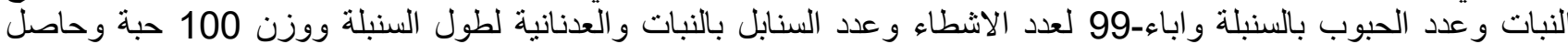

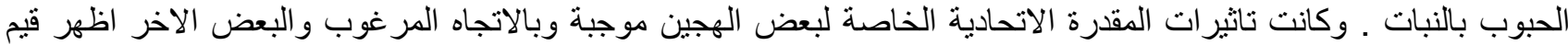

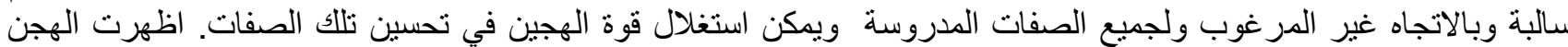

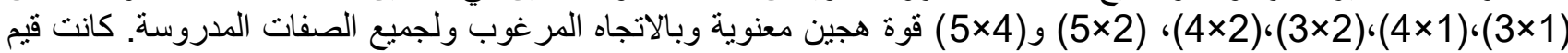

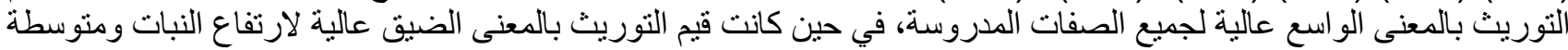

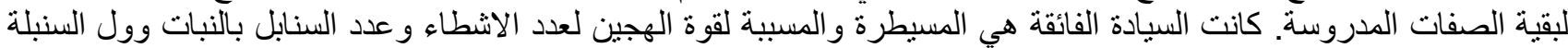
و عدد الحبوب بالسنبلة ووزن 100 حبة وحاصل الحبوب بالنبات، في حين كانت السيادة الجزئية هي المسيطرة و المسببة لقوة الهجين

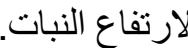

الكلمات المفتاحية: التهجينات التبادلية، المقدرة الاتحادية، قوة الهجين، التوريث ، معدل درجة السيادة، الحنطة الناعمة.

المقدمة: Introduction

تكمن أهمية الحنطة الناعمة (حنطة الخبز) في كونها من أهم محاصيل الحبوب زراعة في العالم فهي تحتل الصدارة في

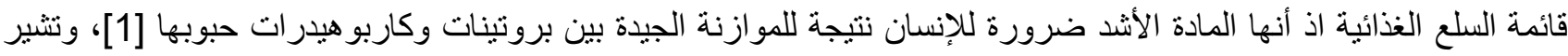

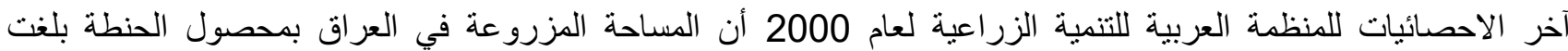

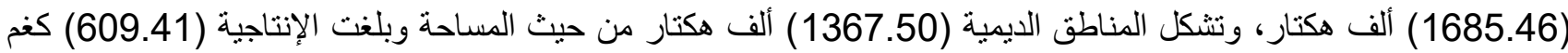

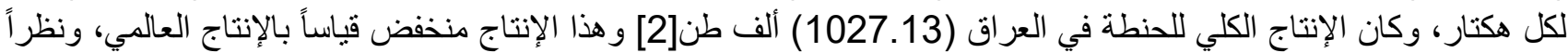

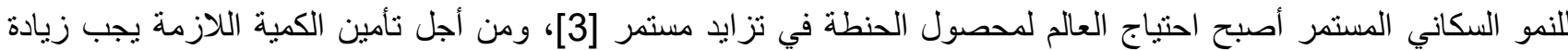

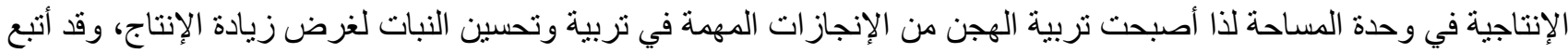
علماء الور اثة وتربية وتحسين النبات طرقاً مختلفة لاختبار الآباء المستخدمة في التهجينات لإنتاج أجيال منعزلة (هجن) ، التينة التي ينتخب

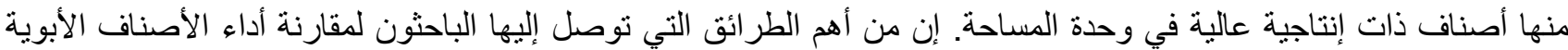

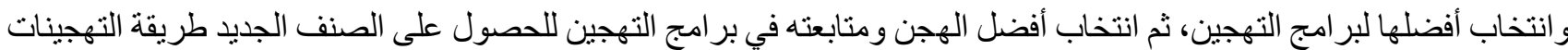

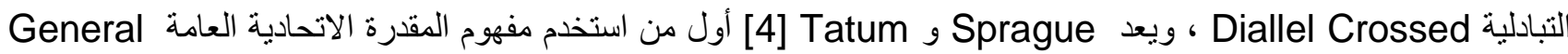
و و المقدرة الاتحادية الخاصة Sombining Ability وبعد ذللك قدم Griffing [5] أربع طر ائق لتحليل التهجينات التبادلية وتقدير المقدرتين الاتحاديتين العامة والخاصة للصفات الكمية

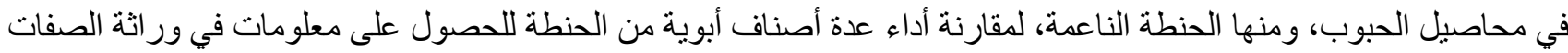

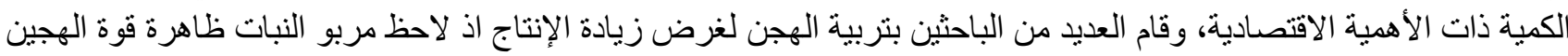

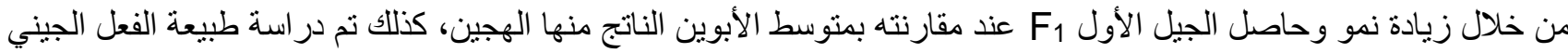

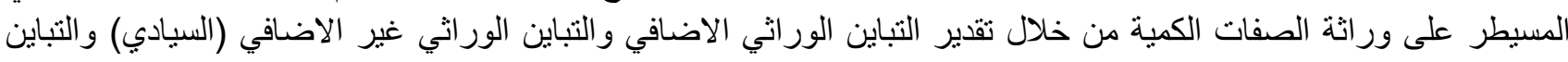

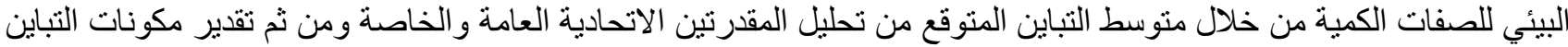

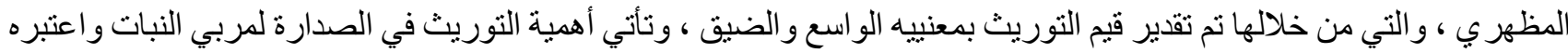

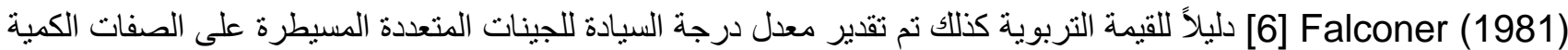
لأنها توضح طبيعة الفعل الجيني للجينات المسيطرة على الصفة الكمية و اعطاء دلالة أفضل على طرق تحسين تلك الصفات الكمية.

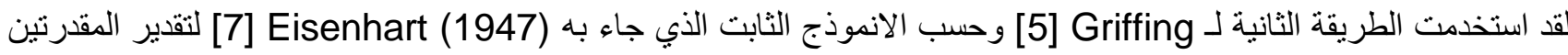

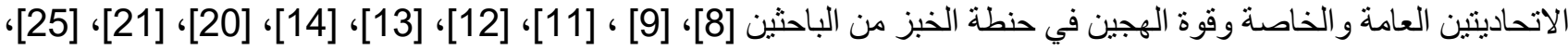

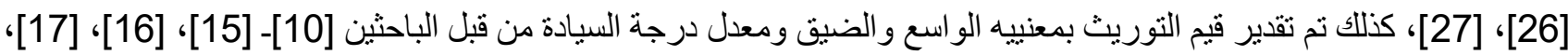
[18]، [19]، [22]، [23]، [24] ـ[28] لدر اسة طبيعة الفعل الجيني المسيطر على وراثة حاصل الحبوب ومكوناته . 
الهدف من الدراسة الحالية تقدير التاثيرات المقدرتين العامة والخاصة على الاتحاد وقوة الهجين وبعض المعالم الور اثية

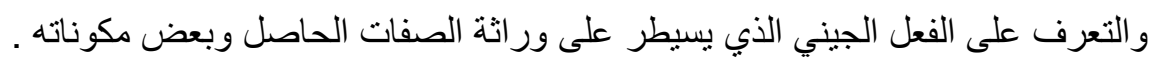

المواد وطر ائق العمل: Material and Methods

الأصناف والتهجينات التبادلية النصفية:

استعملت في هذه الدر اسة خمسة أصناف من الحنطة الناعمة (مسجلة معتمدة في العراق) ونقية ور اثثياً كآباء وهي (تموز

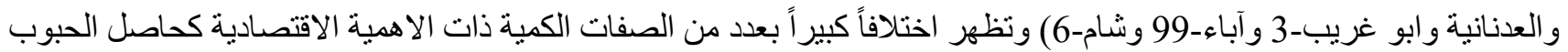

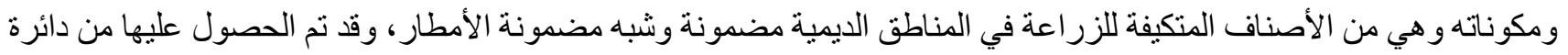

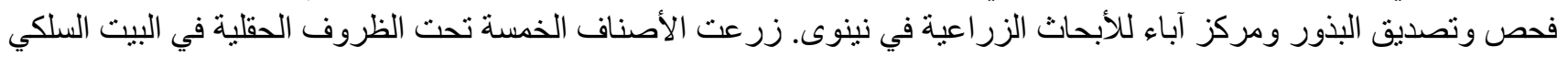

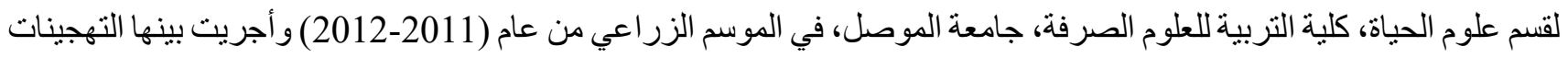

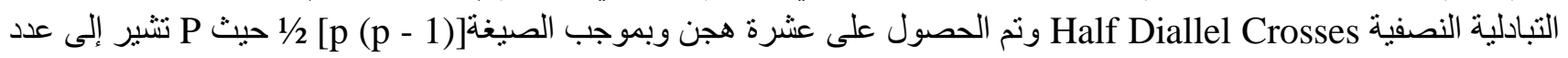
الأصناف الابوية.

\section{التصميم التجريبي والصفات الكمية المدروسة:}

زرعت حبوب التراكيب الور اثية الخمسة عشرة والتي نثمل الأصناف الخمسة الابوية والهجن التبادلية النصفية العشرة

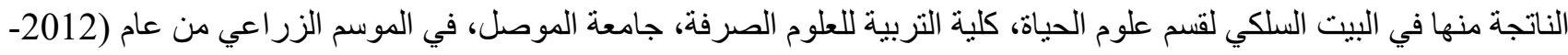

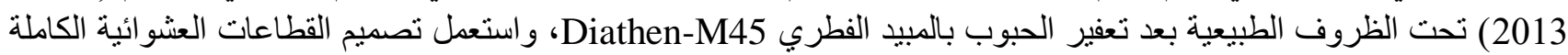

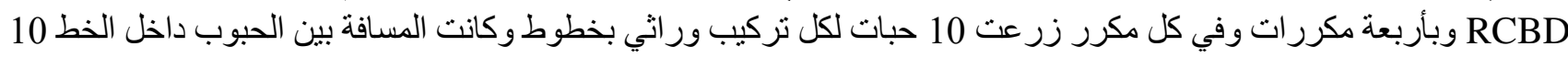

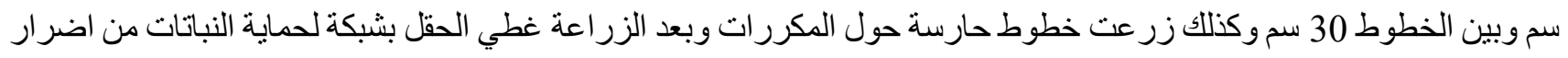

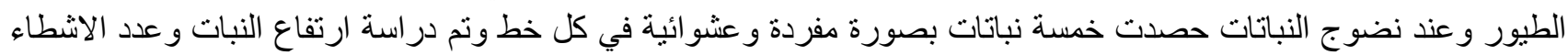

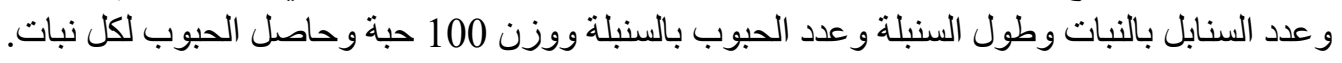

تحليل التباين والمقدرة الاتحادية:

اجري تحليل التباين لكل صفة حسب تصميم القطاعات العشو ائية الكاملة والانموذج الثابت (باعتماد خمسة مشاهدات في كل

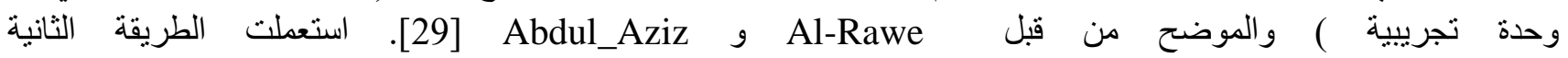

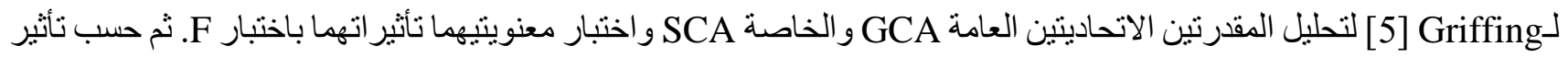

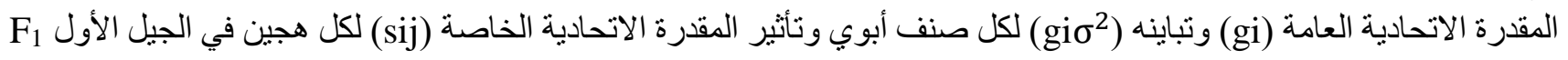

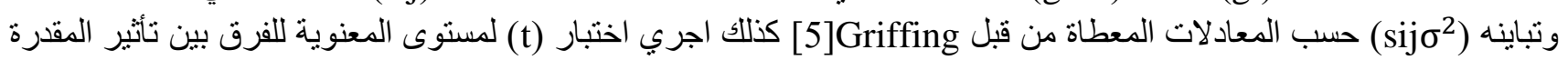

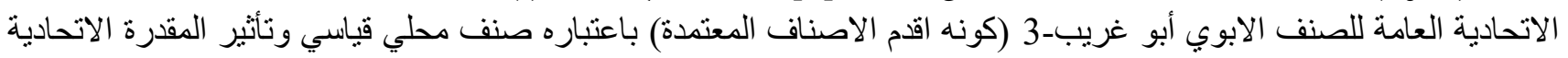

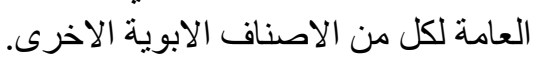
تقدير قوة الهجين وبعض الثوابت الوراثية: قدرت قوة الهجين (H) للصفات الكمية المدروسة على اساس انحر اف منوسط الهجين عن معدل الابوين وبموجب المعادلة

$$
H=\bar{F}-\frac{\overline{P_{i}}+\overline{P_{j}}}{2}
$$

اذ أن $\bar{P}_{i j}$ منوسط الجيل الأول الناتج من تهجين i و ز.

تم حساب تباين قوة الهجين بحساب قيمة t لكل هجين بالمعادلة الاتية:

$$
V(H)=V \bar{F}_{i}+\frac{1}{4}\left[V \bar{P}_{i}+V \bar{P}_{j}\right]
$$


واختبرت معنوية قوة الهجين (H) بحساب قيمة t لكل هجين بالمعادلة الاتية :

$$
t=\frac{H}{\sqrt{V H}}
$$

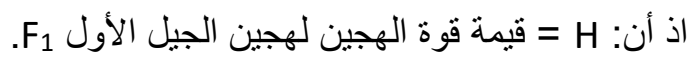

$$
\text { F⿸ }
$$

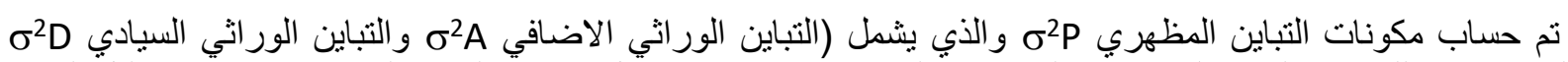

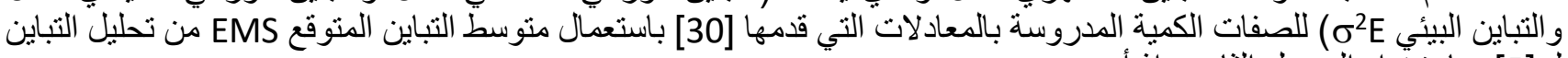
لـ [5] وباستخدام الموديل الثابت، الذّات أن:

$$
\begin{gathered}
\sigma^{2} A=2\left(\sigma^{2} G C A\right) \\
\sigma^{2} D=\left(\sigma^{2} S C A\right) \\
\sigma^{2} E=\left(\sigma^{2} \text { Error }\right)
\end{gathered}
$$

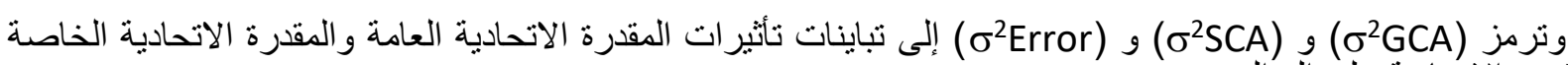
وخطأ المقدرة الاتحادية على التو والي.

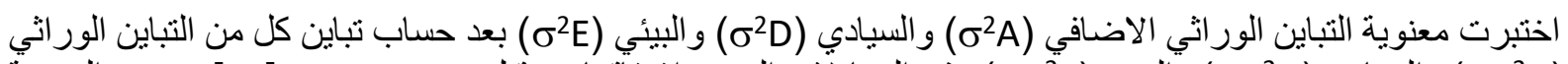

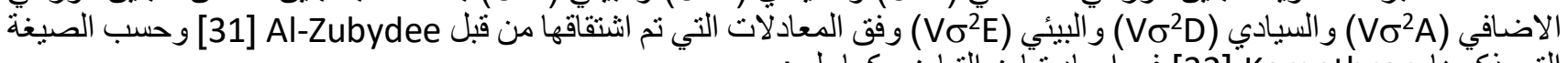

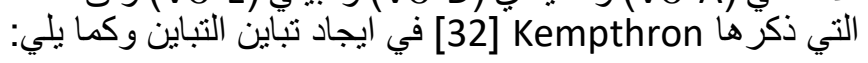

$$
\begin{gathered}
V \sigma^{2} A=\frac{4}{r^{2}(p+2)^{2}}\left[\frac{2(M s G C A)^{2}}{K+2}+\frac{2\left(M \operatorname{ses}^{\prime}\right)^{2}}{K+2}\right] \\
\sigma^{2} D=\frac{1}{r^{2}}\left[\frac{2(M S S C A)^{2}}{K+2}+\frac{2\left(M s e s^{\prime}\right)^{2}}{K+2}\right] \\
\sigma^{2} E=\frac{2 M \operatorname{ses}^{\prime 2}}{K+2}
\end{gathered}
$$

$$
\text { اذ أن: r = عدد المكررات، p= عدد الأصناف الابوية. }
$$

= متوسط المربعات المتوقعة للمقدرة الاتحادية العامة من جدول تحليل التباين.

= متوسط المربعات المتوقعة للمقدرة الاتحادية الخاصة من جدول تحليل التباين.

= منوسط المربعات المنوقعة لخطأ المقدرة الاتحادية من جدول تحليل التباين و المساوي للتباين البيئي. درجات الحرية لكل مصدر من مصادر التباين الواردة في المعادلات. K

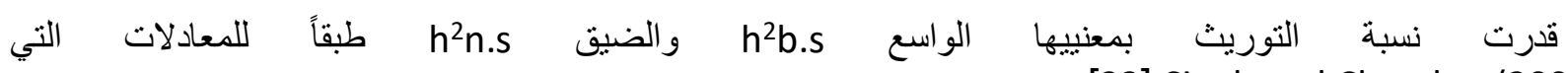

قدمها (2007) Singh and Chaudary

$$
\begin{gathered}
100 \times h^{2} \text { b.s }=\frac{\sigma^{2} G}{\sigma^{2} P}=\frac{\sigma^{2} A+\sigma^{2} D}{\sigma^{2} A+\sigma^{2} D+\sigma^{2} E} \\
100 \times h^{2} \text { n.s }=\frac{\sigma^{2} A}{\sigma^{2} P}==\frac{\sigma^{2} A}{\sigma^{2} A+\sigma^{2} D+\sigma^{2} E}
\end{gathered}
$$

اذ أن:

$$
\begin{array}{r}
\left(+\sigma^{2} D \sigma^{2} A\right)=\text { يمنتل التباين الوراثي: } \sigma^{2} G \\
\left(+\sigma^{2} D+\sigma^{2} E \sigma^{2} A\right)=\text { يمثل التباين المظهري : } \sigma^{2} P
\end{array}
$$




$$
\begin{aligned}
& \text { نم التعبير عن قيم التوريث بالمعنى الواسع (h2b.s) ضمن النسب الآتية: } \\
& \text { أقل من 40\% و واطئة ومن 40\%-60\% منوسطة وأكثر من 60\% عالية [35]. ونم التعبير عن قيم التوريث بالمعنى } \\
& \text { الضيق (h²n.s) ضمن النسب الآتية: }
\end{aligned}
$$

أقل من 20\% واطئة ومن 20\%-50\% منوسطة وأكثر من 50\% عالية [34]. حسب معدل درجة السيادة (a)) وفق المعادلة التي قدمهامobinson واخرون [36].

$$
\begin{gathered}
\bar{a}=\sqrt{\frac{2 \sigma^{2} D}{\sigma^{2} A}} \\
\text { اذ أن: }{ }^{(\bar{a})}
\end{gathered}
$$

النتائج والمناقشة: Results and Discussion

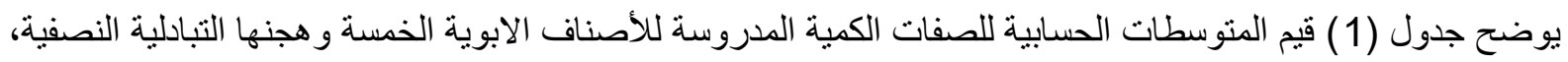

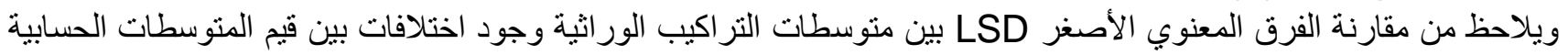

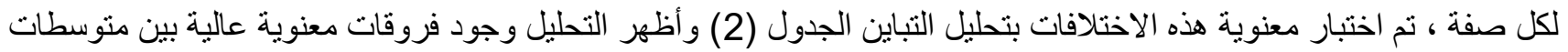

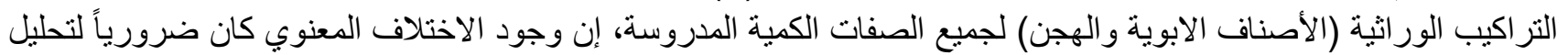

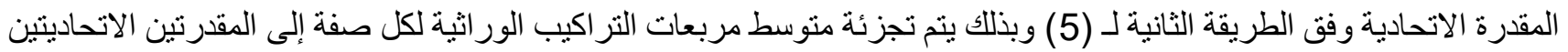

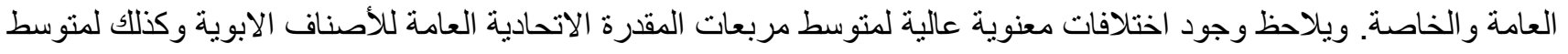

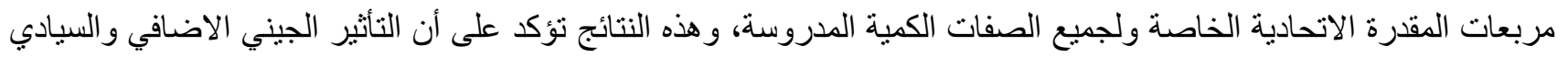

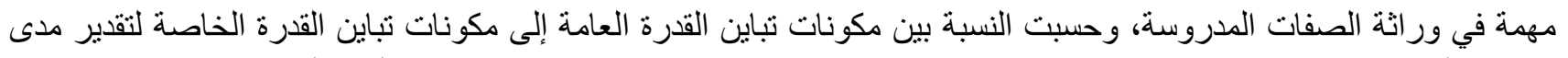

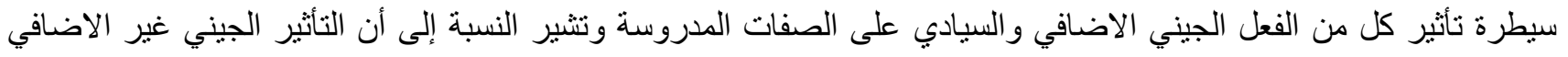

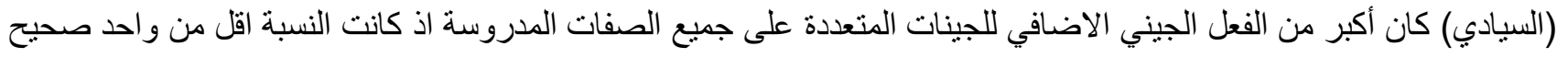

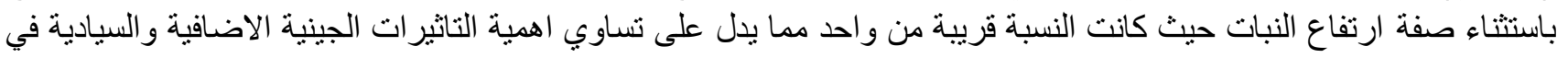

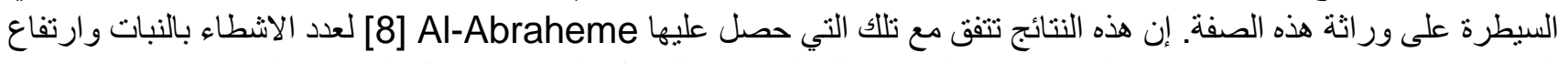

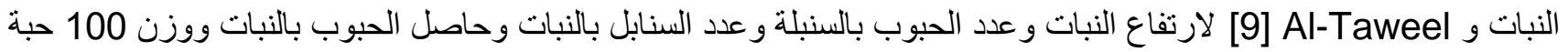

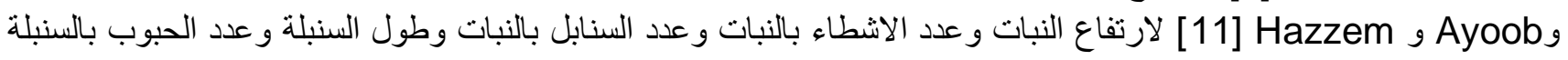

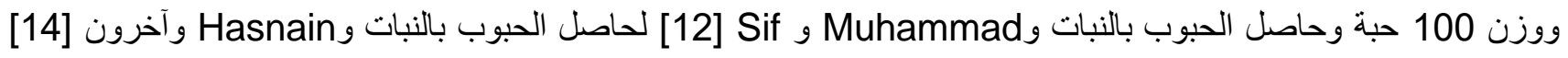

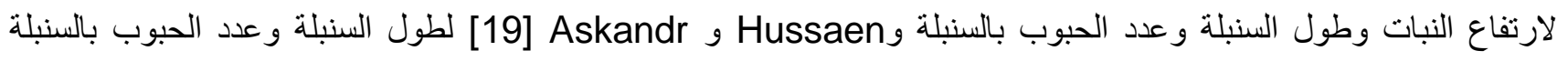

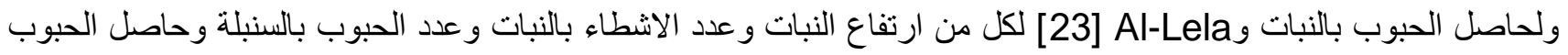

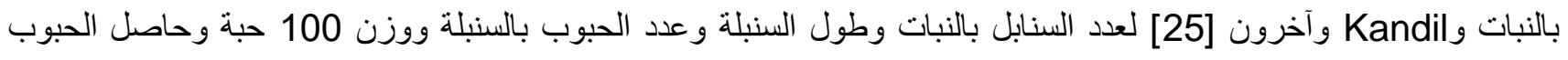

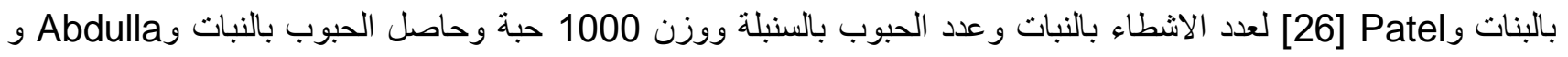

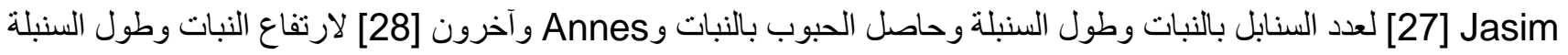
وحاصل الحبوب بالنبات.

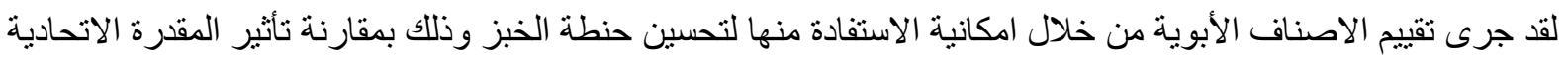

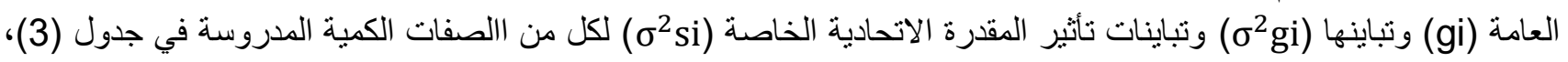

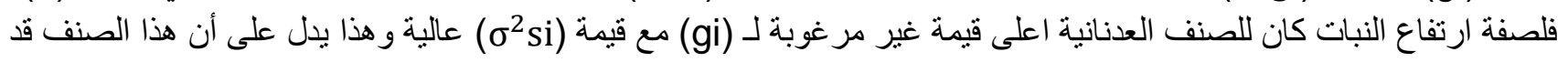

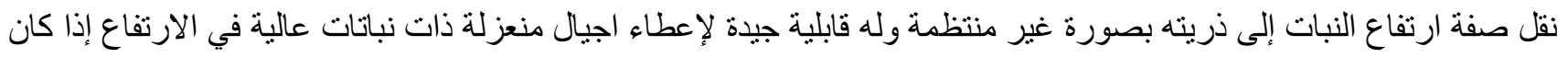

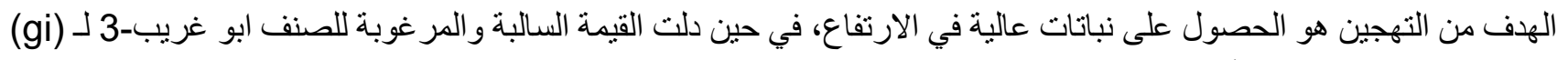

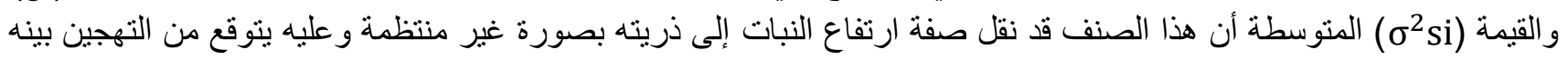

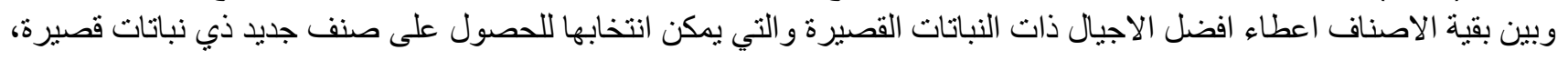

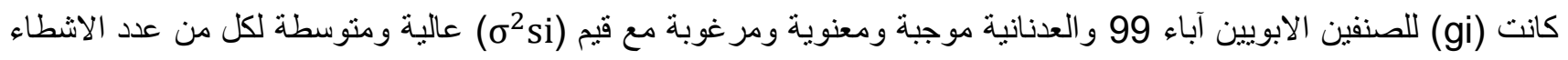

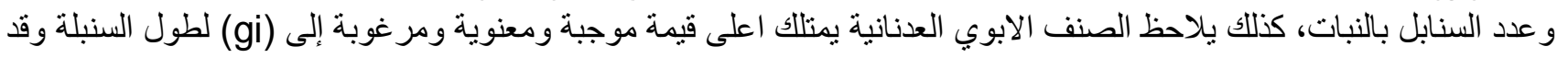




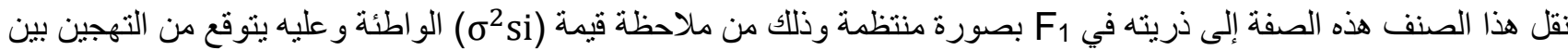

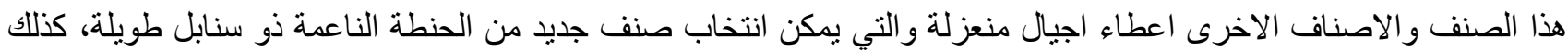

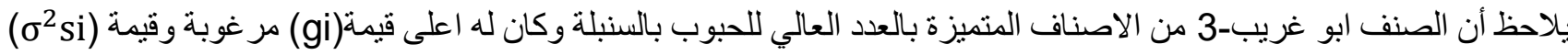

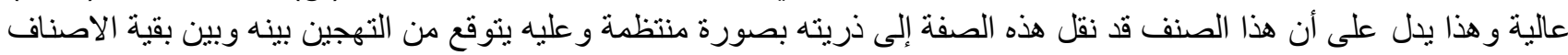

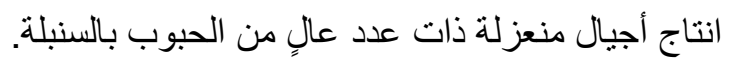

جدول (1): قيم المتوسطات الحسابية للصفات الكمية المدروسة وللتراكيب الوراثية من الحنطة الناعمة

\begin{tabular}{|c|c|c|c|c|c|c|c|}
\hline بالنبات (غبو) & وزبة (غم) 100 & عالسنبلة & طول السنبلة & بالنبات & عالنبات الاشطاء & النبات (سم) & التراثية \\
\hline 13.95 & 3.62 & 35.22 & 9.86 & 5.75 & 6.52 & 91.77 & 1 \\
\hline 19.62 & 4.30 & 46.31 & 11.98 & 7.82 & 8.41 & 92.25 & 2 \\
\hline 12.76 & 3.15 & 37.10 & 9.30 & 4.89 & 5.80 & 84.33 & 3 \\
\hline 14.49 & 4.10 & 45.13 & 11.22 & 6.14 & 6.75 & 82.65 & 4 \\
\hline 18.34 & 3.85 & 41.11 & 10.69 & 7.12 & 7.83 & 78.33 & 5 \\
\hline 16.95 & 4.10 & 38.22 & 11.52 & 7.25 & 7.91 & 90.57 & $2 \times 1$ \\
\hline 15.11 & 3.92 & 35.40 & 9.14 & 8.20 & 8.54 & 82.75 & $3 \times 1$ \\
\hline 16.23 & 3.76 & 32.82 & 10.92 & 9.10 & 9.50 & 95.46 & $4 \times 1$ \\
\hline 13.62 & 3.92 & 39.50 & 11.53 & 5.93 & 6.69 & 89.54 & $5 \times 1$ \\
\hline 16.35 & 4.13 & 42.62 & 10.95 & 7.60 & 7.71 & 92.81 & $3 \times 2$ \\
\hline 19.59 & 4.83 & 52.18 & 12.88 & 9.15 & 9.61 & 96.30 & $4 \times 2$ \\
\hline 14.36 & 4.75 & 50.17 & 11.44 & 7.72 & 8.70 & 83.79 & $5 \times 2$ \\
\hline 15.97 & 3.56 & 48.13 & 10.15 & 5.43 & 6.61 & 84.40 & $4 \times 3$ \\
\hline 13.94 & 3.33 & 46.10 & 11.20 & 6.38 & 7.25 & 78.66 & $5 \times 3$ \\
\hline 18.15 & 4.41 & 56.29 & 13.12 & 7.95 & 8.82 & 82.54 & $5 \times 4$ \\
\hline 2.29 & 0.32 & 3.62 & 0.54 & 0.29 & 0.75 & 4.73 & L.S.D. \\
\hline
\end{tabular}

تمثل الأرقام 1، 2، 3، 4، 5 الأصناف الأبوية: تموز-3 والعدنانية وابو غريب-3 و آباء-99 وشام-6 على التو الي.

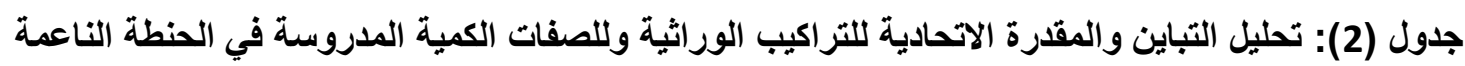

\begin{tabular}{|c|c|c|c|c|c|c|c|c|}
\hline 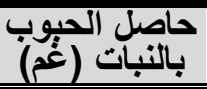 & وزن 100 حبه & عالدينبّة & طول السنبله & عالد السنابل & عدالآنشات & ارتقاع النبات & الدرجاتّ & مصادر التباين \\
\hline 246.85 & 14.86 & 678.25 & 2.75 & 65.78 & 89.85 & 883.75 & 3 & \\
\hline$* * 121.64$ & $* * 12.19$ & $* * 408.17$ & $* * 12.28$ & $* * 36.25$ & $* * 45.16$ & **319.86 & 14 & \\
\hline 27.86 & 4.52 & 115.21 & 3.12 & 4.22 & 5.88 & 40.82 & 42 & طا التجريب \\
\hline 20.32 & 1.04 & 50.22 & 0.26 & 2.88 & 3.11 & 20.35 & 240 & طا العين \\
\hline$* * 28.46$ & $* * 1.58$ & $* * 92.89$ & $* * 1.18$ & $* * 1.82$ & $* * 2.88$ & **126.36 & 4 & \\
\hline **10.84 & $* * 0.74$ & *33.40 & $* * 0.56$ & $* * 0.79$ & $* * 1.56$ & $* * 18.62$ & 10 & قدرة الاتحادية \\
\hline 1.42 & 0.04 & 2.66 & 0.03 & 0.13 & 0.16 & 3.51 & 42 & لخط \\
\hline 0.41 & 0.33 & 0.42 & 0.13 & 0.35 & 0.28 & 1.16 & & 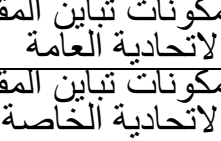 \\
\hline
\end{tabular}


تشير النتائج في جدول (3) ايضاً أن الصنفين الابويين العدنانية وآباء-99 كان لها قيمة (gi) عالية معنوية ومر غوبة لوزن الهن

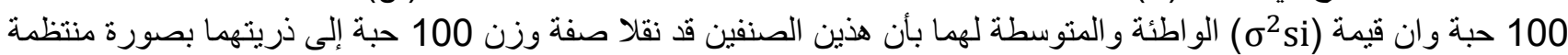

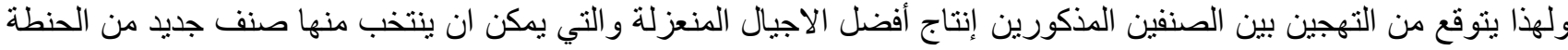

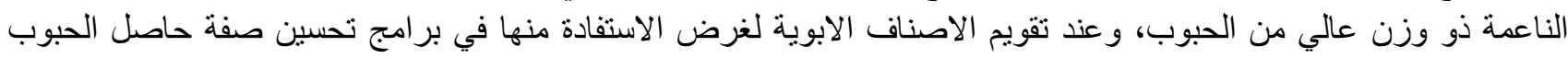

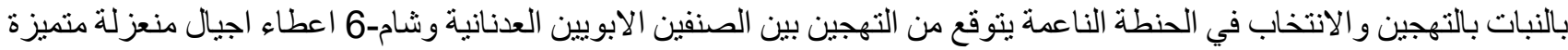

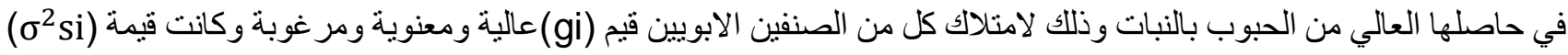

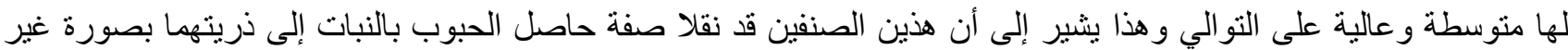

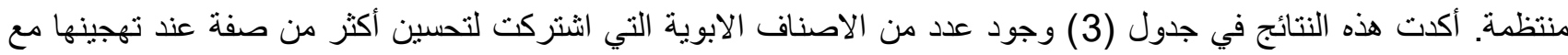

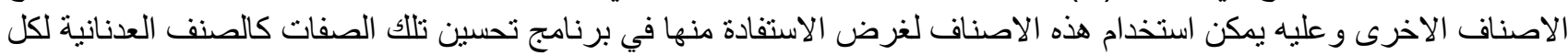

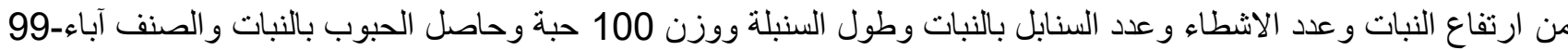

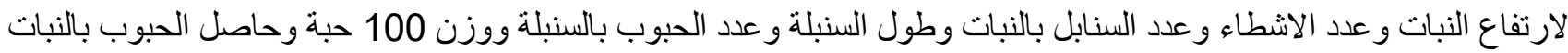

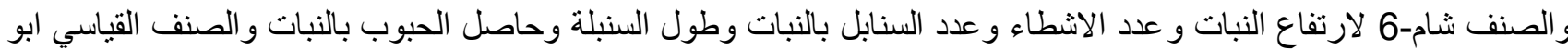

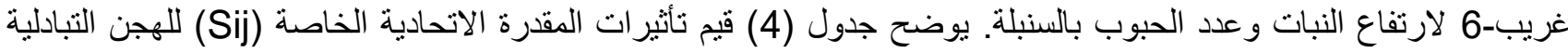

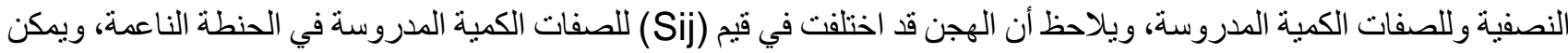
الاستفادة من الهجن التي تمتلك قيم (Sij) مرغوبة في برامج تحسين الصفات الكمية باستغلال قوة الهجين ويلاحظ الكان (5) (5) هجناً

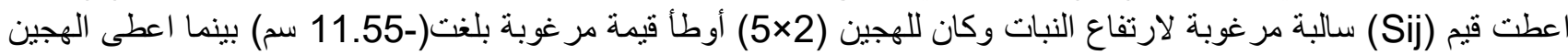

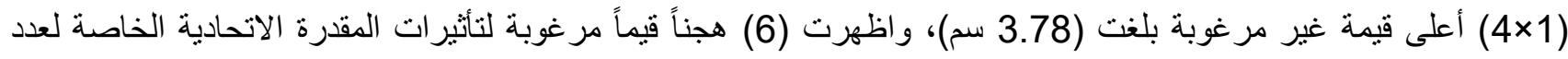

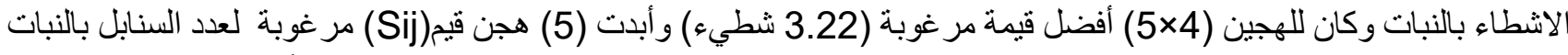

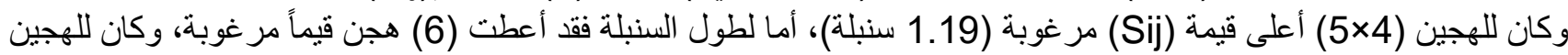

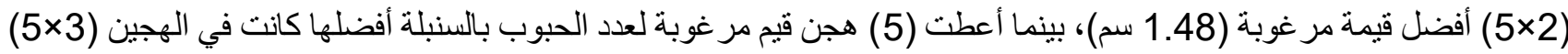

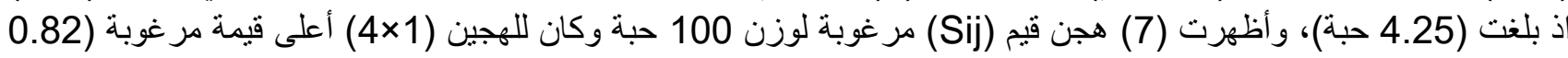

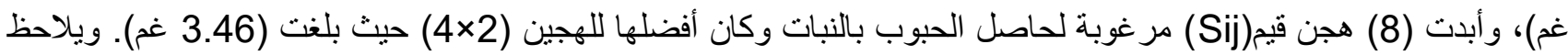

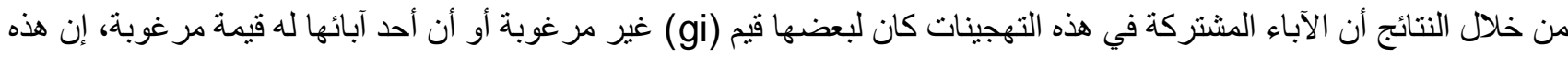

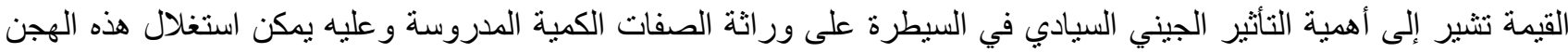

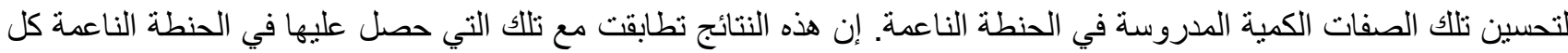

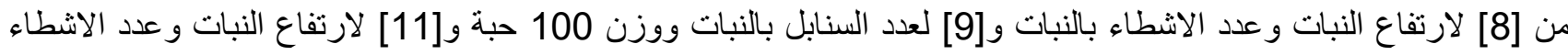

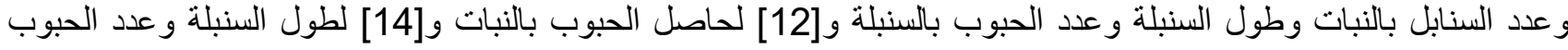

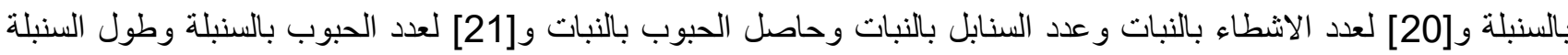

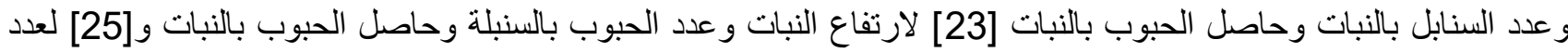

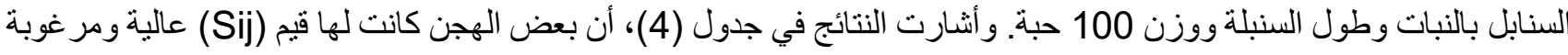

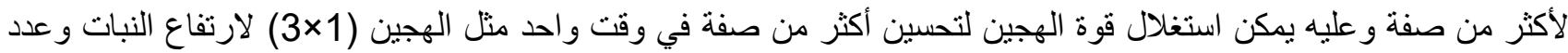

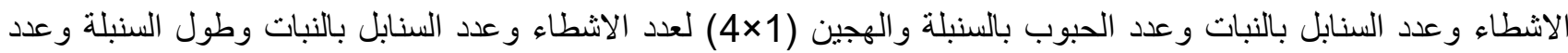

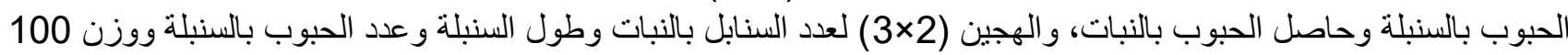

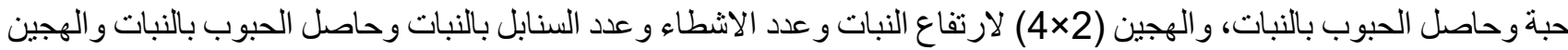

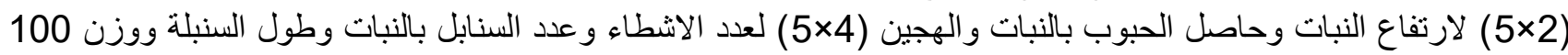

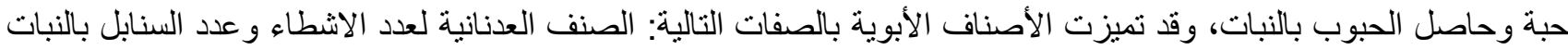

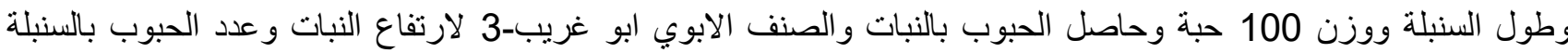

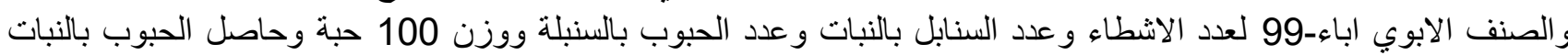

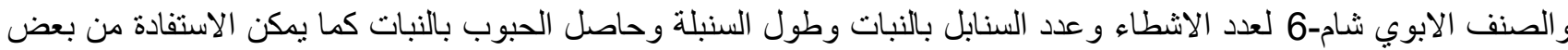

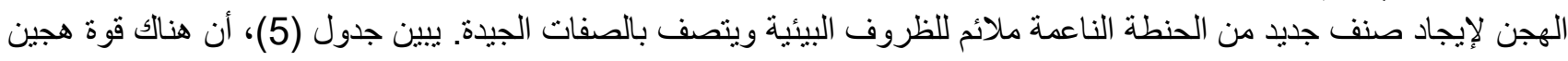

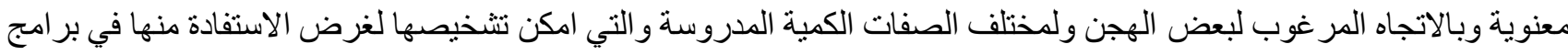

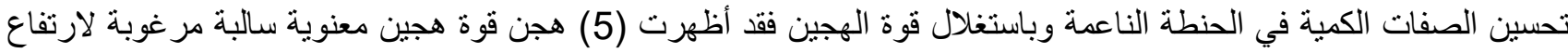

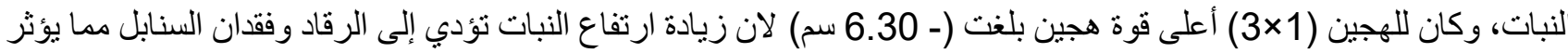
على حاصل الحبوب الكلي للنبات و عليه يمكن الاستفادة من الهجن التي أبدت قوة هجين سالبة ومعنوية لارتفاع الناع النبات لألنات النها الهدف 


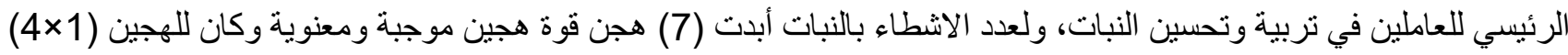

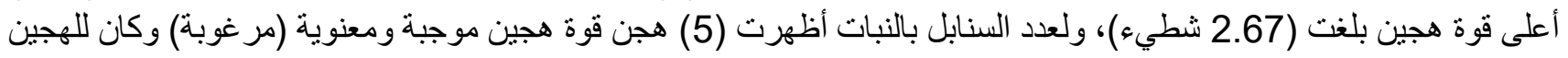

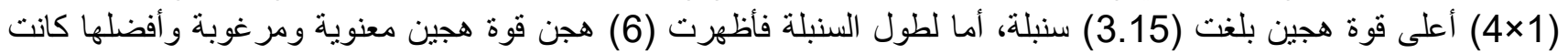

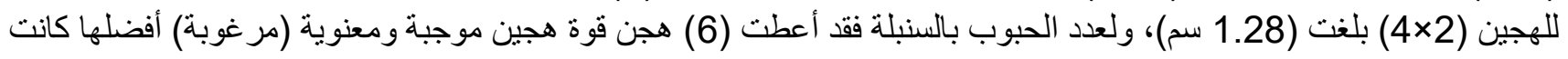

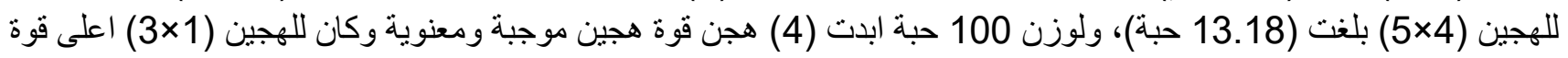

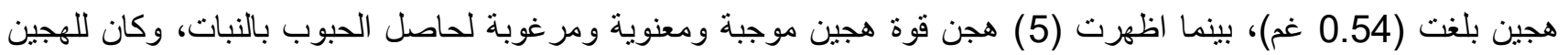

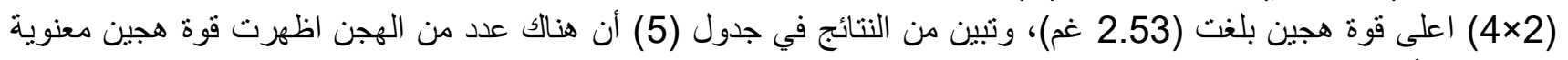

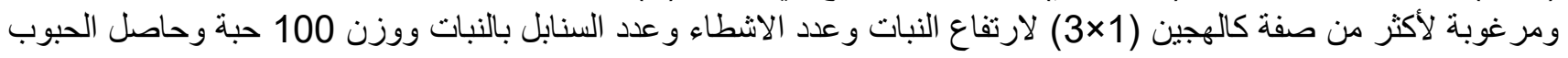

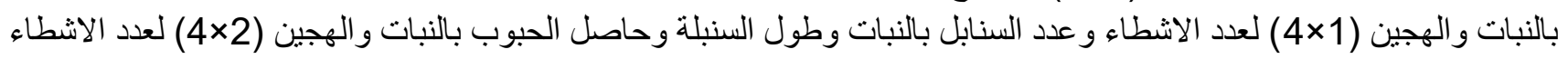

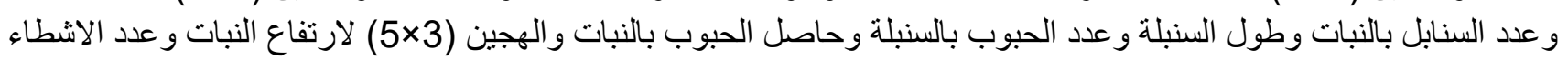

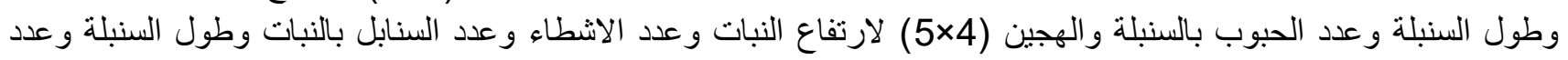

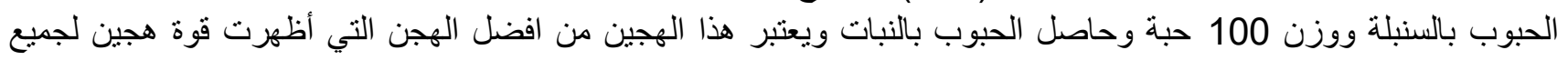

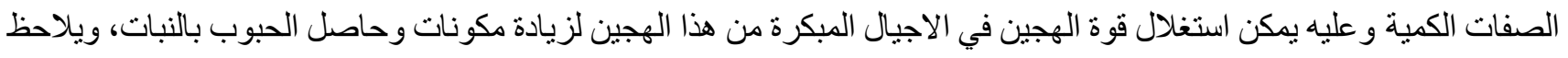

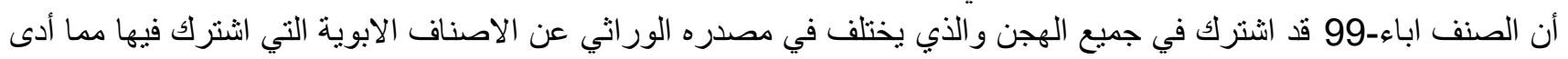

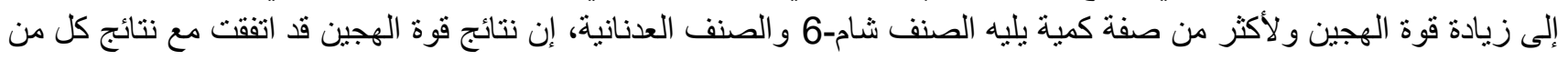

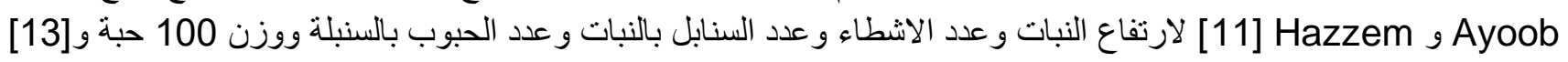

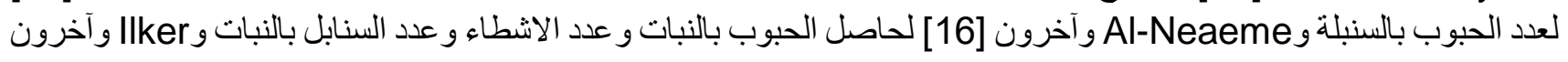

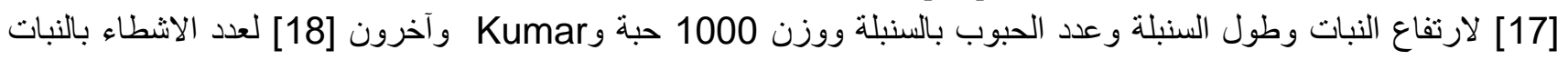

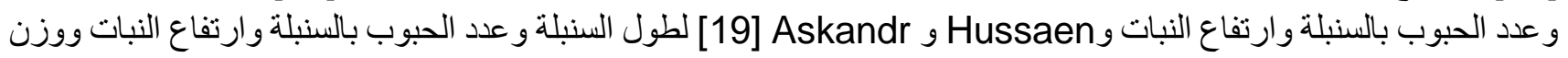

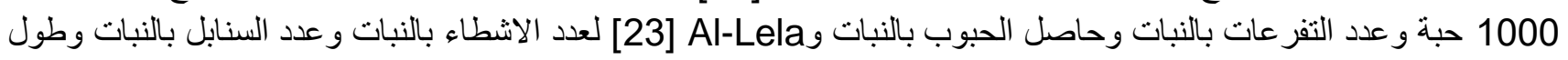

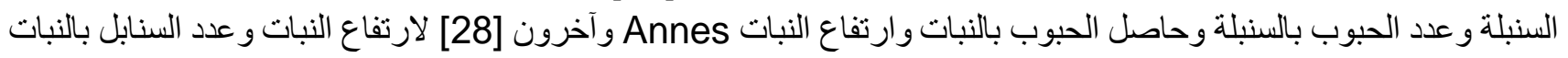

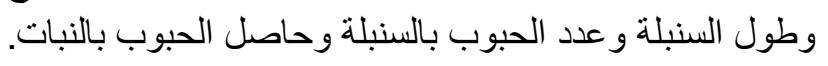




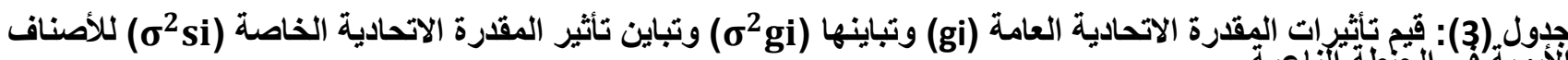

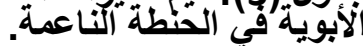

\begin{tabular}{|c|c|c|c|c|c|c|c|c|}
\hline 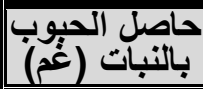 & وزن 100 حبه & بالسنبئلة الحوب & طول السنبلَ) & عالالنبات السنابل & عدالالنبات & ارتقاع) النبات & والتباتينيات ات & الالاصيوية \\
\hline $\begin{array}{c}2.06- \\
1.005 \\
0.84\end{array}$ & $\begin{array}{c}* * 0.13 \\
0.04 \\
0.03\end{array}$ & $\begin{array}{c}1.10 \\
0.53 \\
18.12\end{array}$ & $\begin{array}{c}* * 0.36- \\
0.21 \\
0.04\end{array}$ & $\begin{array}{c}* * 0.48- \\
0.14 \\
20.15\end{array}$ & $\begin{array}{l}0.36- \\
0.20 \\
3.12\end{array}$ & $\begin{array}{c}* * 2.08 \\
1.67 \\
6.32\end{array}$ & $\begin{array}{c}\text { gi } \\
\sigma^{2} \mathrm{gi} \\
\sigma^{2} \mathrm{Si}\end{array}$ & موز-3 \\
\hline $\begin{array}{l}* * 07 \\
1.25 \\
1.21\end{array}$ & $\begin{array}{l}* * 2.16 \\
0.01\end{array}$ & $\begin{array}{c}* * 2.11- \\
0.33 \\
5.62\end{array}$ & $\begin{array}{c}* * 0.75 \\
0.66\end{array}$ & $\begin{array}{l}* * 0.46 \\
0.15 \\
205 ?\end{array}$ & $\begin{array}{c}* * 0.54 \\
0.16 \\
870\end{array}$ & $\begin{array}{l}* * 4.14 \\
21.37 \\
3025\end{array}$ & $\sigma_{2 i}^{g i}$ & \\
\hline $\begin{array}{l}1.14 \\
1.23- \\
3.11 \\
0.89\end{array}$ & $\begin{array}{l}0.05 \\
0.21- \\
0.04 \\
0.06\end{array}$ & $\begin{array}{c}* * 4.26 \\
6.44 \\
15.87\end{array}$ & $\begin{array}{l}0.31- \\
0.07 \\
0.05\end{array}$ & $\begin{array}{c}0.56- \\
0.48 \\
22.13\end{array}$ & $\begin{array}{l}0.64- \\
0.53 \\
0.06\end{array}$ & $\begin{array}{c}* * 7.52- \\
19.57 \\
4.32\end{array}$ & $\underset{\sigma^{2}}{\sigma_{\mathrm{Si}}^{2}}$ & بو غريب-3 \\
\hline $\begin{array}{c}* * 0.36 \\
0.12- \\
0.78\end{array}$ & $\begin{array}{c}* * 1.85 \\
0.002- \\
0.09\end{array}$ & $\begin{array}{c}* * 3.50 \\
6.96 \\
13.42\end{array}$ & $\begin{array}{l}* * 0.52 \\
0.06 \\
0.20\end{array}$ & $\begin{array}{c}* * 0.66 \\
0.32 \\
1766\end{array}$ & $\begin{array}{c}* * 1.14 \\
0.99 \\
1058\end{array}$ & $\begin{array}{l}* * 1.56 \\
0.84 \\
0.5\end{array}$ & $\sigma_{2}^{g i}$ & باء-99 \\
\hline $\begin{array}{c}* * 1.12 \\
0.28 \\
3.55\end{array}$ & $\begin{array}{c}* * 0.05- \\
0.003 \\
0.04\end{array}$ & $\begin{array}{c}1.13- \\
0.88 \\
17.83\end{array}$ & $\begin{array}{l}* * 0.21 \\
0.04 \\
0.42\end{array}$ & $\begin{array}{l}{ }^{* *} 0.08 \\
0.01- \\
25.41\end{array}$ & $\begin{array}{l}* * 0.09 \\
0.02- \\
1.03\end{array}$ & $\begin{array}{l}* * 0.99 \\
0.88- \\
22.56\end{array}$ & $\sigma_{\sigma^{2}} \mathrm{gi}_{\mathrm{si}}^{2}$ & ن فنام-6 \\
\hline 0.25 & 0.01 & 0.37 & 0.08 & 0.11 & 0.13 & 0.48 & $\begin{array}{l}\text { S.E(gi- } \\
\text { gs) }\end{array}$ & \\
\hline
\end{tabular}

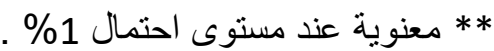

جدول (4): تقدير تأثيرات المقدرة الاتحادية الخاصة (Sij) للهجن التبادلية النصفية وللصفات الكمية المدروسة في الحنطة الناعمة.

\begin{tabular}{|c|c|c|c|c|c|c|c|}
\hline 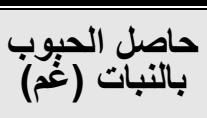 & وزن (غم) 100 حبة & بالسنبلّة بوب & طول السنبلة & عدالنبات السنابل & عدالاتبات & النباتئ & الهجن \\
\hline 0.27 & $0.08-$ & $0.60-$ & $0.18-$ & $0.35-$ & $0.25-$ & 2.18- & $2 \times 1$ \\
\hline $1.42-$ & $0.31-$ & 2.09 & $0.02-$ & 0.55 & 0.77 & 1.89- & $3 \times 1$ \\
\hline $0.15-$ & 0.82 & 4.85- & 0.43 & 0.87 & 1.32 & 3.78 & $4 \times 1$ \\
\hline 1.48 & 0.04 & 3.79- & 0.12 & $0.58-$ & 2.14 & 1.22 & $5 \times 1$ \\
\hline 2.37 & 0.29 & 2.47 & 0.59 & 0.21 & $0.15-$ & 1.68 & $3 \times 2$ \\
\hline 3.46 & $0.37-$ & $0.38-$ & $0.37-$ & 0.94 & 0.88 & $0.89-$ & $4 \times 2$ \\
\hline 1.35 & 0.62 & 0.91 & 1.48 & 0.63 & 0.97- & $11.55-$ & $5 \times 2$ \\
\hline 0.69 & 0.06 & 0.93 & $0.18-$ & $0.81-$ & 0.01- & 1.31 & $4 \times 3$ \\
\hline $1.58-$ & 0.07 & 4.25 & 0.22 & $0.42-$ & 0.33 & 3.12- & $5 \times 3$ \\
\hline 0.17 & 0.14 & 0.96- & 0.62 & 1.19 & 3.22 & 0.78 & $5 \times 4$ \\
\hline 0.29 & 0.08 & 1.14 & 0.15 & 0.09 & 0.62 & 1.73 & S.E(Sik-Sij) \\
\hline
\end{tabular}

تمثل الأرقام 1، 2، 3، 4، 5 الأصناف الأبوية: تموز-3 و العدنانية وابو غريب-3 وآباء-99 وشام-6 على التوالي. 
جدول (5): قوة الهجين للصفات الكمية المدروسة في الهجن الناتجة من التهجينات التبادلية النصفية بين خمسة أصناف أبوية من الحنطة الناعمة وعلى أساس معدل الأبوين.

\begin{tabular}{|c|c|c|c|c|c|c|c|}
\hline بالنبات (غِم) & وزن 100 حبة & عالد الحبنّب & طول السنبلة & عالنبات السنابل & عداد الاشطاء & ارتفاع النبات & لأصناف الأبوية \\
\hline $\begin{array}{c}0.16 \\
0.08 \pm\end{array}$ & $\begin{array}{c}0.14 \\
0.17 \pm\end{array}$ & $\begin{array}{c}* * 2.55- \\
0.16 \pm\end{array}$ & $\begin{array}{l}* * 0.60 \\
0.04 \pm\end{array}$ & $\begin{array}{c}0.45 \\
0.37 \pm\end{array}$ & $\begin{array}{l}* * 0.60 \\
0.13 \pm\end{array}$ & $\begin{array}{c}* * 2.44- \\
0.21 \pm\end{array}$ & $2 \times 1$ \\
\hline $\begin{array}{l}* * 1.75 \\
0.13 \pm\end{array}$ & $\begin{array}{l}* * 0.54 \\
0.03 \pm\end{array}$ & $\begin{array}{c}0.76 \\
0.45 \pm\end{array}$ & $\begin{array}{l}0.44- \\
0.32 \pm\end{array}$ & $\begin{array}{l}* * 2.83 \\
0.21 \pm\end{array}$ & $\begin{array}{l}* * 2.38 \\
0.21 \pm\end{array}$ & $\begin{array}{c}* * 6.30- \\
0.26 \pm\end{array}$ & $3 \times 1$ \\
\hline $\begin{array}{l}* * 2.01 \\
0.45 \pm\end{array}$ & $\begin{array}{c}0.10- \\
0.08 \pm\end{array}$ & $\begin{array}{l}* * 7.36 \\
1.02 \pm\end{array}$ & $\begin{array}{l}* * 0.41 \\
0.03 \pm\end{array}$ & $\begin{array}{l}* * 3.15 \\
0.22 \pm\end{array}$ & $\begin{array}{l}* * 2.67 \\
0.18 \pm\end{array}$ & $\begin{array}{l}* * 7.25 \\
1.11 \pm\end{array}$ & $4 \times 1$ \\
\hline $\begin{array}{c}* * 2.54- \\
0.35 \pm\end{array}$ & $\begin{array}{l}* * 0.19 \\
0.04 \pm\end{array}$ & $\begin{array}{c}1.33 \\
0.66 \pm\end{array}$ & $\begin{array}{l}* * 1.26 \\
0.12 \pm\end{array}$ & $\begin{array}{l}0.51- \\
1.81 \pm\end{array}$ & $\begin{array}{l}0.19- \\
1.05 \pm\end{array}$ & $\begin{array}{l}* * 3.38 \\
0.25 \pm\end{array}$ & $5 \times 1$ \\
\hline $\begin{array}{c}0.16 \\
0.07 \pm\end{array}$ & $\begin{array}{l}* * 0.41 \\
0.07 \pm\end{array}$ & $\begin{array}{l}* 3.10- \\
0.78 \pm\end{array}$ & $\begin{array}{c}0.31 \\
0.17 \pm\end{array}$ & $\begin{array}{c}* * 1.19- \\
0.21 \pm\end{array}$ & $\begin{array}{l}0.76- \\
1.62 \pm\end{array}$ & $\begin{array}{l}* * 4.52 \\
0.35 \pm\end{array}$ & $3 \times 2$ \\
\hline $\begin{array}{l}* * 2.53 \\
0.19 \pm\end{array}$ & $\begin{array}{c}* * 0.63- \\
0.08 \pm\end{array}$ & $\begin{array}{l}* * 8.46 \\
1.22 \pm\end{array}$ & $\begin{array}{l}* * 1.28 \\
0.21 \pm\end{array}$ & $\begin{array}{l}* * 2.17 \\
0.23 \pm\end{array}$ & $\begin{array}{l}* * 2.18 \\
0.55 \pm\end{array}$ & $\begin{array}{l}* * 2.85 \\
0.41 \pm\end{array}$ & $4 \times 2$ \\
\hline $\begin{array}{l}* * 4.62- \\
0.89 \pm\end{array}$ & $\begin{array}{c}0.68 \\
0.29 \pm\end{array}$ & $\begin{array}{l}* * 6.41 \\
0.02 \pm\end{array}$ & $\begin{array}{c}0.11 \\
0.12 \pm\end{array}$ & $\begin{array}{l}* * 1.23 \\
0.10 \pm\end{array}$ & $\begin{array}{l}* * 2.25 \\
0.42 \pm\end{array}$ & $\begin{array}{l}1.16- \\
0.75 \pm\end{array}$ & $5 \times 2$ \\
\hline $\begin{array}{l}* * 2.34 \\
0.52 \pm\end{array}$ & $\begin{array}{c}0.07- \\
0.03 \pm\end{array}$ & $\begin{array}{l}* * 7.13 \\
0.25 \pm\end{array}$ & $\begin{array}{l}0.10- \\
0.31 \pm\end{array}$ & $\begin{array}{c}0.09- \\
0.08 \pm\end{array}$ & $\begin{array}{l}0.12- \\
0.17 \pm\end{array}$ & $\begin{array}{c}0.91 \\
1.05 \pm\end{array}$ & $4 \times 3$ \\
\hline $\begin{array}{c}* * 1.61- \\
0.17 \pm\end{array}$ & $\begin{array}{l}0.17- \\
0.12 \pm\end{array}$ & $\begin{array}{l}* * 6.99 \\
0.55 \pm\end{array}$ & $\begin{array}{l}* * 1.20 \\
0.14 \pm\end{array}$ & $\begin{array}{l}0.37- \\
0.35 \pm\end{array}$ & $\begin{array}{l}* * 0.73 \\
0.08 \pm\end{array}$ & $\begin{array}{c}* * 2.78- \\
0.43 \pm\end{array}$ & $5 \times 3$ \\
\hline $\begin{array}{l}* * 1.73 \\
0.30 \pm\end{array}$ & $\begin{array}{l}* * 0.44 \\
0.07 \pm\end{array}$ & $\begin{array}{c}* * 13.18 \\
0.16 \pm\end{array}$ & $\begin{array}{l}* * 2.17 \\
0.25 \pm\end{array}$ & $\begin{array}{l}* * 1.25 \\
0.14 \pm\end{array}$ & $\begin{array}{l}* * 1.13 \\
0.12 \pm\end{array}$ & $\begin{array}{c}* * 1.39- \\
0.17 \pm\end{array}$ & $5 \times 4$ \\
\hline
\end{tabular}

تمثل الأرقام 1، 2، 3، 4، 5 الأصناف الأبوية: تموز-3 والعدنانية وابو غريب-3 و اباء-99 وشام-6، على التو الي.

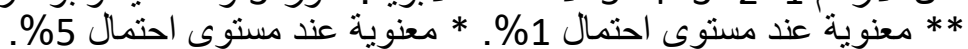

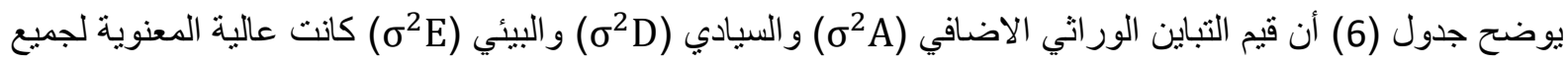

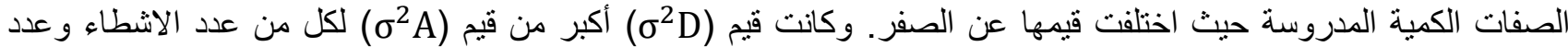

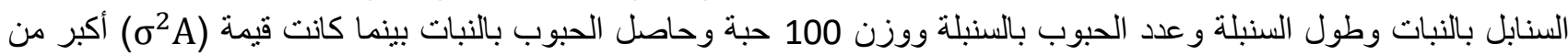

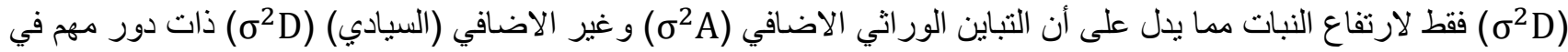

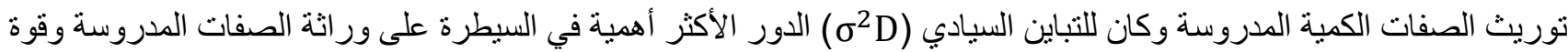

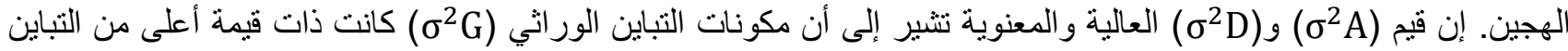

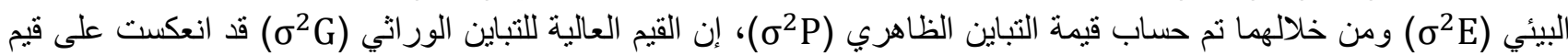
نسبة التوريث بمعنييه الواسع والضيق ونأتي أهمية التوريث في الصدارة لمربي النبات، كانت قيمة التوريث بالمباتين المعنى الواسع عالية

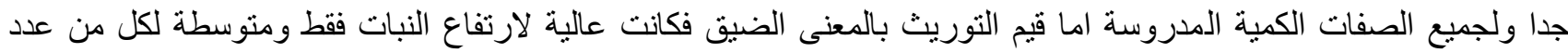
الاشطاء و عدد السنابل بالنبات وطول السنبلة و عدد الحبوب بالسنبلة ووزن 100 حبة وحاصل الحبوب بالنبات التبات و انعكست قيم التباين

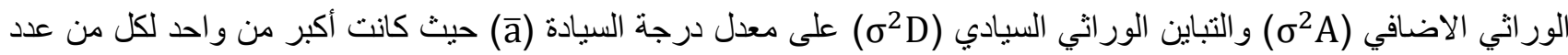

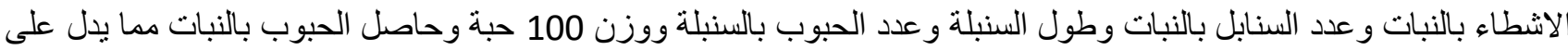


وجود السيادة الفائقة (over dominance) لأغلب الجينات المتعددة المسيطرة على وراثة هذه الصفات في حين كان معدل درجة

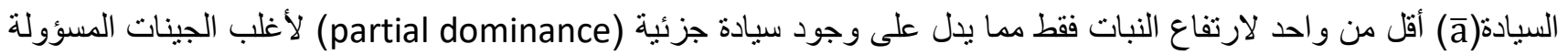

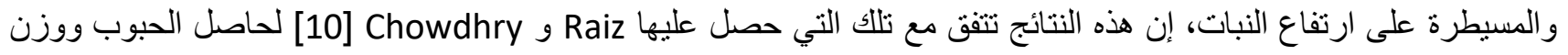

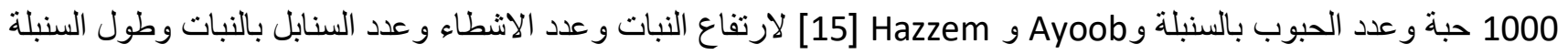

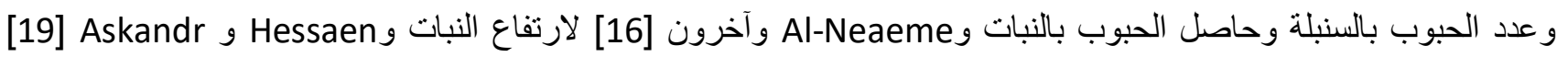

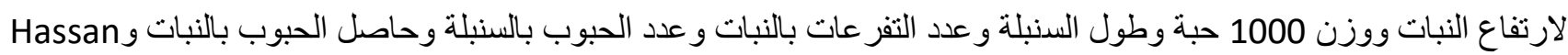

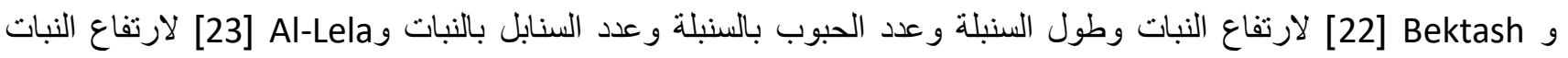

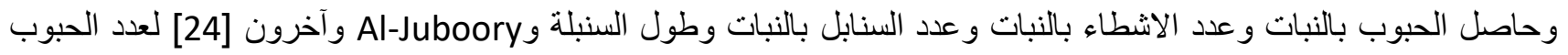

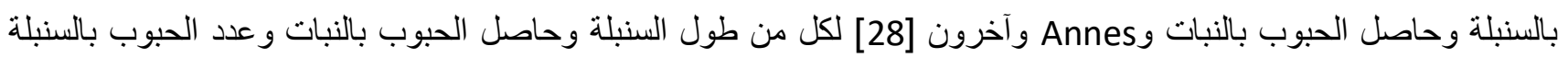
و عدد السنابل بالنبات.

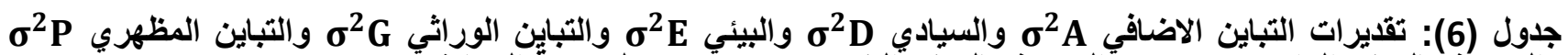

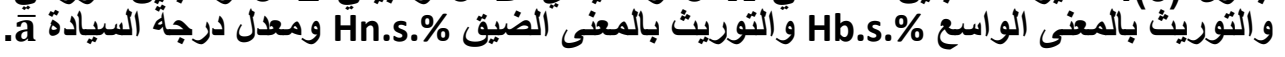

\begin{tabular}{|c|c|c|c|c|c|c|c|}
\hline حالنبات الَبوب & وزن 100 حبة & عالسنبلة الحبوب & طول السنبلة & عدالنبات السنابل & عدالنبات الاشطاء & 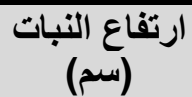 & الثوابت \\
\hline $\begin{array}{l}* * 1.94 \\
0.12 \pm\end{array}$ & $\begin{array}{l}* * 0.12 \\
0.03 \pm\end{array}$ & $\begin{array}{l}* * 6.44 \\
0.06 \pm\end{array}$ & $\begin{array}{l}* * 0.08 \\
0.01 \pm\end{array}$ & $\begin{array}{l}* * 0.12 \\
0.02 \pm\end{array}$ & $\begin{array}{l}* * 0.20 \\
0.01 \pm\end{array}$ & $\begin{array}{l}* * 8.76 \\
1.30 \pm\end{array}$ & $\sigma^{2} \mathrm{~A}$ \\
\hline $\begin{array}{l}* * 2.36 \\
0.58 \pm\end{array}$ & $\begin{array}{l}* * 0.18 \\
0.03 \pm\end{array}$ & $\begin{array}{l}* * 7.69 \\
0.13 \pm\end{array}$ & $\begin{array}{l}* * 0.13 \\
0.02 \pm\end{array}$ & $\begin{array}{l}* * 0.17 \\
0.03 \pm\end{array}$ & $\begin{array}{l}* * 0.35 \\
0.04 \pm\end{array}$ & $\begin{array}{l}* * 3.78 \\
0.54 \pm\end{array}$ & $\sigma^{2} \mathrm{D}$ \\
\hline $\begin{array}{l}* * 1.42 \\
0.18 \pm\end{array}$ & $\begin{array}{l}* * 0.04 \\
0.001 \pm\end{array}$ & $\begin{array}{l}* * 2.66 \\
0.32 \pm\end{array}$ & $\begin{array}{l}* * 0.03 \\
0.004 \pm\end{array}$ & $\begin{array}{l}* * 0.13 \\
0.01 \pm\end{array}$ & $\begin{array}{l}* * 0.16 \\
0.02 \pm\end{array}$ & $\begin{array}{l}* * 3.51 \\
0.40 \pm\end{array}$ & $\sigma^{2} \mathrm{E}$ \\
\hline 4.30 & 0.30 & 14.13 & 0.21 & 0.29 & 0.55 & 12.54 & $\sigma^{2} \mathrm{G}$ \\
\hline 5.72 & 0.34 & 16.79 & 0.45 & 0.42 & 0.71 & 16.05 & $\sigma^{2} \mathrm{P}$ \\
\hline 80.11 & 88.23 & 84.16 & 87.50 & 69.05 & 76.06 & 78.13 & $\% h^{2}$ b.s. \\
\hline 27.17 & 35.29 & 38.37 & 33.33 & 28.57 & 28.17 & 54.58 & $\% h^{2}$ n.s. \\
\hline 1.56 & 1.73 & 1.55 & 3.25 & 1.68 & 1.87 & 0.92 & $\overline{\mathrm{a}}$ \\
\hline
\end{tabular}

\% * * معنوية عند مستوى احتمال

Conclusion الاستنتاجات

اظهرت نتائج در اسة تحليل المقدرة الاتحادية وتقدير قوة الهجين وبعض المعالم الور اثية باستعمال التهجين التبادلي النصفي في حنطة الخبز ما يأتي:

(1) معنوية عالية لمتوسط مربعات المقدرتين الاتحاديتين العامة والخاصة ولجميع الصفات المدروسة وتبين ان كلا التاثيرين الجينيين

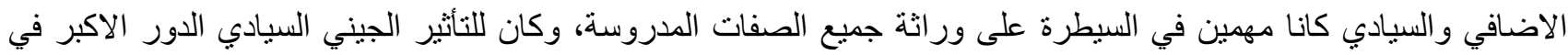

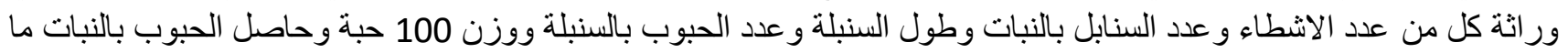

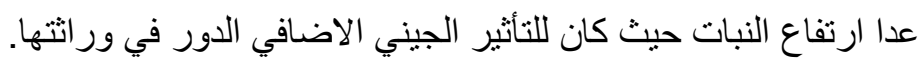

(2) امكانية الحصول على معلومات حول اداء الاصناف الابوية الخمسة ذات القيمة العالية والمعنوية لتأثير المقدرة الاتحادية العامة

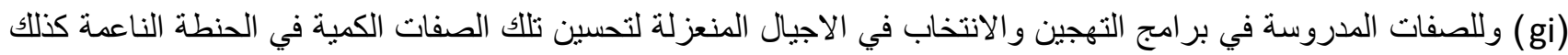

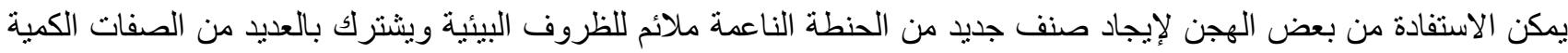


من خلال قيم تأثثر المقدرة الاتحادية الخاصة (Sij).(3) اظهرت بعض الهجن قوة هجين معنوية وبالاتجاه المرغوب لكل من الصفات

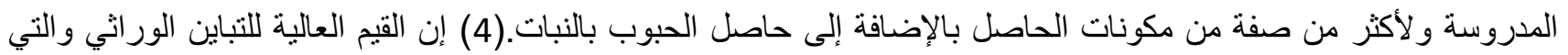

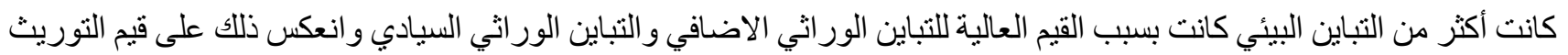

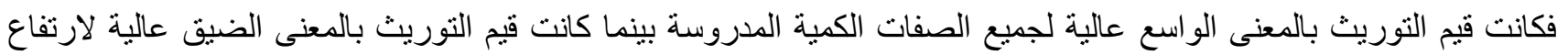

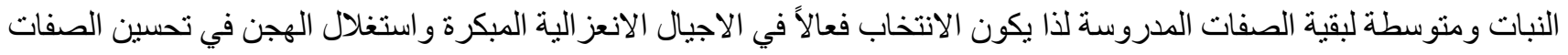

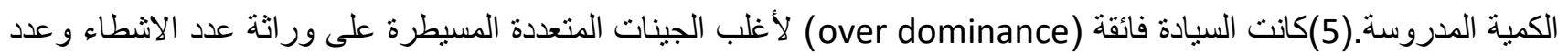

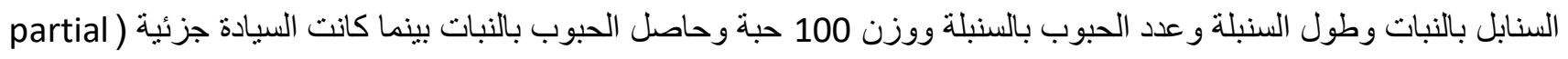

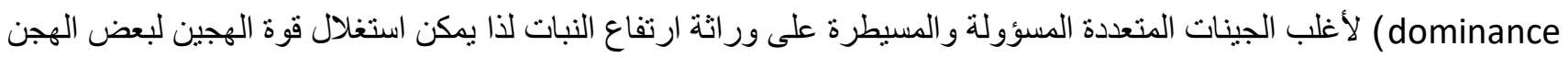

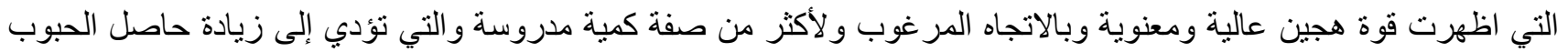
بالنبات.

References المصادر

1- Wilsie C.P. "Crop Adiptation and Distribution" Freeman W.H. Company San Francisco. (1962).

2- Arab Agricultural statistics year book, Arab organization for agricultural development , league of Arab states, Alkhartoum, December 20:26-76(2000).

3- Rajaram S. "Prospests and Promise of wheat Bread in the $21^{\text {st }}$ century" wheat conf. Budapest-Hungar (2000).

4- Spraque G.F. and Tatum. L.A., J.Amer. Soc. Agron., 34: 923-932. (1942).

5- Griffing B., Aust.J. Biol., Sci. 9: 463-493 (1956).

6- Falconer D.S. "Introduction to Quantitative Genetics" Longman Group limited London (1981).

7- Eisenhart C., Biometrics, 3: 1-12 (1947).

8- Al-Abraheme. S.M. M.Sc. Thesis, College of Science, University of Mosul (2000). (In Arabic).

9- Al-Taweel . M.S.M. M.Sc. Thesis, College of Agriculture and forestay , University of Mosul (2003). (In Arabic).

10- Raiz R.. and Chowdhry M.S. Asian S. of Plant Sciences 2(10): 748-755 (2003).

11- Ayoob M.H. and Hazzem A.N., Rafidain Journal of Science 16(2): 173-183(2006).

12- Muhammad I. and Sif A., International J. of Agric. And Bio., (5) : 688-690 (2006).

13- Esra A.c. and Koksal Y., Tarim. Bilimleri. Drgisis, 13(4): 354-364 (2007).

14- Hasnain Z., Abbas G., Saeed A., Shakell A., Muhammad A. and Rahim M.A., MiddlleEast J. Sci. Res 2:128-131 (2007). 
15- Ayoob M.H. and HazzemA.N., Journal of Educ. And Sci. special volume of the first conference on Biology, September 20(2):342-348(2007).

16- Al-Neaeme J.J., Al-Khafajy H.M. and Sarheed A.F., Alforat Journal of Agriculture sciences 1(4): 157-162(2009).

17- Ilker E., Tonk P.A., and Tosun M., Pak.J. Bot. 42(1): 513-522 (2010).

18- Kumar A., Mishra V.K., Vyas R.P. and Singh V., Journal of plant Breeding and Crop Science 3(10): 209-217 (2011).

19- Hussaen M.A. and Askandr H.S, , Mesoptamia Journal of Agriculture 39(2):132141(2011).

20- Ali A.H., Mesopotamia Journal of Agriculture 40(16):66-72 (2012).

21- Adel M.M. and Ali M.M., Asian Journal of Crop Seience 5(1): 14-23 (2013).

22- Hassan L.K. and Bektash F.Y., the Iraqi Journal of Agriculture Science 45(8):822835(2014).

23- Al-Lela M.J., Jordan Journal of Agriculture Sciences 11(2): 507-524(2015).

24- Al-Juboory A.H., Dawood S.M. Al-Obaiidy and Jasim M. Al- Juboory, Diyala Agriculture Science Jurnal, 8(2): 13-27 (2016).

25- Kandil A.A., Sharief A.E. and Hasnaa S.M.G., Inter .J. Agro. And Agric. Res. 8(2) 37-44. (2016).

26- patel H.N., Electronic Journal of Plant Breeding, 8(2): 404-408 (2017).

27- Abdulla A.H. Jasim A.H., Tikrit university Journal of Agriculture Sciences17(1):1222(2017).

28-Annes, A.H., Al-Zubaydee K.M.D., Al-Obaiidy D.S.M. and Al-Obaiidy S.A.E., Annals Journal of Agriculture Sciences Moshtohor 56(1):113-122(2018).

29- Al-Rawe K.M. and Abdul_Aziz M.K.A., " Design and analysis of agricultural experiments " Dar-Akutub and publishing directors, Mosul university , Iraq (2000)

30- Hallauev A.R. and Miranda J.B. "Quantitative Genetic in Maize Breeding". Lowa State Univ. Press, Ames. Lowa, Chapter 4, 52-64(1981).

31- Al-Zubaydee,K.M.D,PH.D, Thesis, college of Agriculture and forestay, University of Mosul (1986).(In Arabic).

32- Kempthorn O. "Introduction to Genetic Statistic" . John Wiley and Sons. New York, Chapter 13, 224-234 (1957). 
33- Singh R.K.. and Chaudary B.D. "Biometrical Methods in Quantitative Genetic Analysis", Rev. Kalyani Puplishers, Ludhiana India (2007).

34- Boho M.N.Doc. Thesis, College of Science, University Mosul (1997). (In Arabic).

35- Al- Athare A.H.M., ' Fundamentals of genetics " second Edition, Dar-Akutub and publishing directors, Mosul university, Iraq (1987).

36- Robinson H.F., Comstok R.E. and Harvey P.H., Agron. J. 41: 353-359 (1949). 